\title{
Estadística oficial y evaluación de políticas públicas: oportunidades y retos
}

\section{Official statistics and public policy evaluation: opportunities and challenges}

\author{
Juan Manuel Rodríguez Póo \\ Antonio Salcedo Galiano \\ Instituto Nacional de Estadística
}

\section{Resumen}

La estadística oficial constituye un bien público al servicio de las sociedades democráticas. La pandemia de la COVID-19 ha demostrado la extraordinaria resiliencia de la estadística, aunque también ha puesto de manifiesto la necesidad de adoptar medidas adicionales orientadas a establecer un mejor uso de las nuevas fuentes de datos, que ofrecen una oportunidad única a la estadística para satisfacer de forma eficiente las crecientes demandas de los usuarios, incrementando la frecuencia y granularidad de la información así como la disponibilidad de nuevos conjuntos de datos, obteniendo una estadística de alta definición en tiempo real que redundaría en un mejor análisis y evaluación de políticas públicas.

Palabras clave: big data, código de buenas prácticas, estadística oficial, evaluación de políticas públicas.

Clasificación JEL: COO, H11.

\begin{abstract}
Official statistics constitute a public good at the service of the democratic societies. The COVID-19 pandemic has shown the extraordinary resilience of statistics, although it has also highlighted the need to take additional measures aimed at making a better use of new data sources, which offer a unique opportunity for statistics to efficiently meet the growing demands of users, increasing the frequency and granularity of the information as well as the availability of new data sets, obtaining high definition statistics in real time that would result in a better analysis and evaluation of public policies.
\end{abstract}

Keywords: big data, code of good practices, official statistics, public policy evaluation. 


\section{Introducción}

La información estadística constituye un elemento de gran importancia en el proceso de evaluación de políticas públicas, aportando la evidencia empírica necesaria para ofrecer una visión precisa de la situación actual, así como para abordar la medición de las relaciones existentes entre las distintas políticas aplicadas y los efectos finalmente alcanzados (Feinstein, 2007). La cada vez mayor utilización de estadísticas en la evaluación de políticas proporciona un singular sustento al proceso de toma de decisiones, reforzando a su vez otros procesos relacionados tales como la transparencia o la rendición de cuentas (Fano, 2017). Este uso cada vez más intensivo de datos estadísticos para evaluación ha ido aparejado con el desarrollo de nuevas técnicas cuantitativas, sistematizadas según los distintos tipos de intervenciones públicas (Moral et al., 2016), junto con la aparición de novedosas formas de acceso a datos y presentación de resultados (UNECE, 2009).

La situación provocada por la pandemia de la COVID-19 ha impulsado aún más la fuerte demanda de estadísticas por parte de los evaluadores de políticas públicas y, de forma general, por la sociedad, en diferentes ámbitos: sociodemográfico, económico, sanitario o de movilidad. Considerando el periodo de tiempo de diez años que abarca desde el 1 de enero de 2011 al 1 de enero de 2021, en la Figura 1 puede apreciarse que los valores máximos de la tendencia de búsqueda de «estadísticas» en España se obtuvieron entre los meses de marzo y abril del año 2020, fechas que precisamente coinciden con el punto álgido de la pandemia. Desde entonces este índice de búsquedas se mantiene en un valor más elevado que la media de años previos.

Sin duda la COVID-19 ha supuesto un extraordinario desafío para la estadística oficial, cuya producción se basaba en buena medida en modelos clásicos de recogida de datos a partir de encuestas (Statistics Canada, 2003), evidenciando la aparición de nuevos y desafiantes retos tecnológicos para la estadística, desde la recogida de datos hasta la propia difusión de la información. Los sistemas estadísticos nacional y europeo han afrontado una crisis sin precedentes y han buscado dar respuesta a dicho desafío, poniendo en marcha nuevos productos estadísticos y publicando información orientada a satisfacer las nuevas demandas de datos, en ocasiones casi en tiempo real.

En este contexto el uso intensivo de los registros administrativos y de forma más general, el big data, han irrumpido con extraordinaria fuerza en la estadística oficial, aportando también importantes beneficios al diseño de políticas públicas, lo que a su vez ha redundado en una mejora de la calidad y la eficiencia de los servicios públicos así como en la más eficiente utilización de recursos (Azzone, 2018). No obstante, el tránsito desde esos grandes volúmenes de datos hacia un conjunto estable de indicadores sintéticos es un camino arduo y complejo que presenta un buen número de dificultades (Arnaboldi \& Azzone, 2020), la primera de las cuales es el propio acceso a esas nuevas fuentes de datos que, en múltiples ocasiones, se encuentran mantenidas por poseedores o tenedores privados de datos. 
FIGURA 1

ÍNDICE DE BÚSQUEDA DE «ESTADÍSTICAS» EN ESPAÑA ENTRE LOS AÑOS 2011-2021

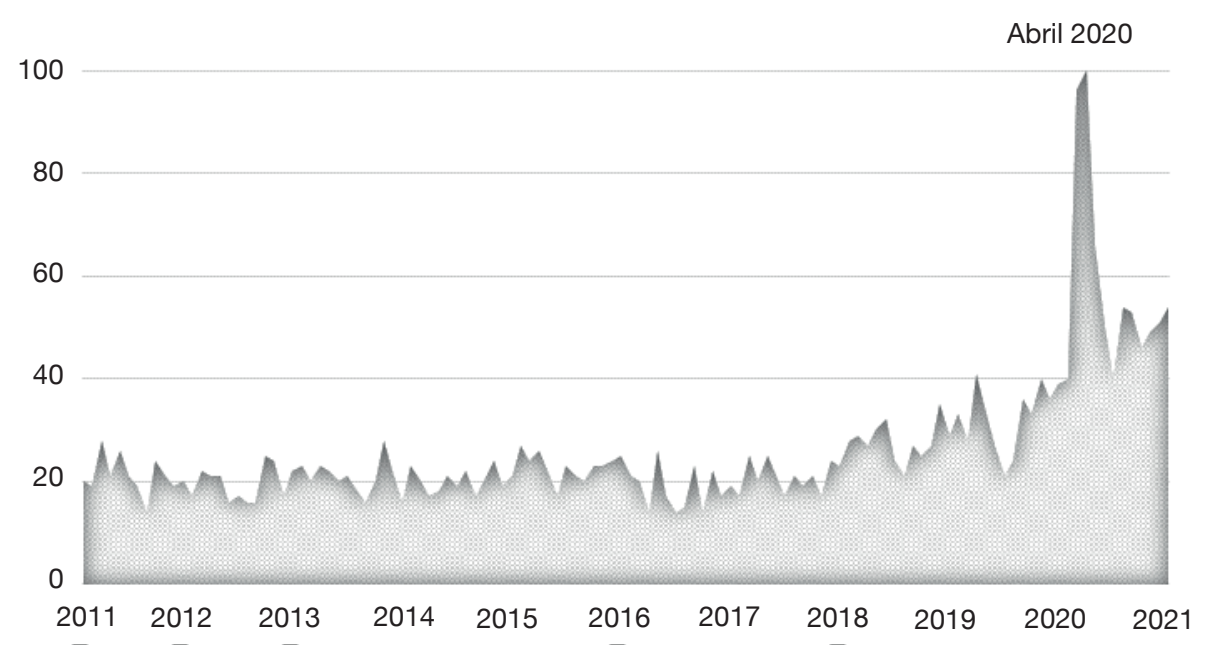

FUENTE: Google Trends y elaboración propia.

En una sociedad altamente datificada y globalizada la estadística oficial debe desempeñar un papel clave en la toma de decisiones basadas en datos (data-driven decision-making) o, de forma más amplia, en el uso de datos para políticas (data for policy, D4P) (Radermacher, 2019). Este rol requiere un estudio detallado de la situación de la estadística oficial en la actualidad bajo la premisa de que este bien público tenga asegurado que podrá disponer de una posición relevante en la definición de las políticas de datos en el corto, medio y largo plazo, facilitando así la continuidad de su desempeño con la máxima eficacia.

\section{Estadísticas, indicadores y evaluación de políticas públicas en España}

Un indicador estadístico constituye una medida resumida relacionada con un problema o fenómeno clave y que se deriva de una serie de hechos observados (Eurostat, 2014). Los conjuntos o sistemas de indicadores constituyen una herramienta clásica a la hora de acometer evaluaciones de políticas públicas. Estos sistemas nos aportan tanto una foto fija de la actuación pública en estudio como su evolución, calculados a partir de los distintos resultados obtenidos. Desde una óptica temporal una política pública puede representarse de forma simplificada de acuerdo con la Figura 2. Esta política pública vendría caracterizada por una posición inicial en un momento $t_{i}$, una trayectoria determinada por los resultados en cada instante temporal $t$ del conjunto de indicadores socioeconómicos seleccionados y, por último, una posición final que sucede en el momento $t_{f}$ (Pollitt \& Bouckaert, 2010). 
FIGURA 2

EVOLUCIÓN DE INDICADORES SOCIOECONÓMICOS DE UNA POLÍTICA PÚBLICA

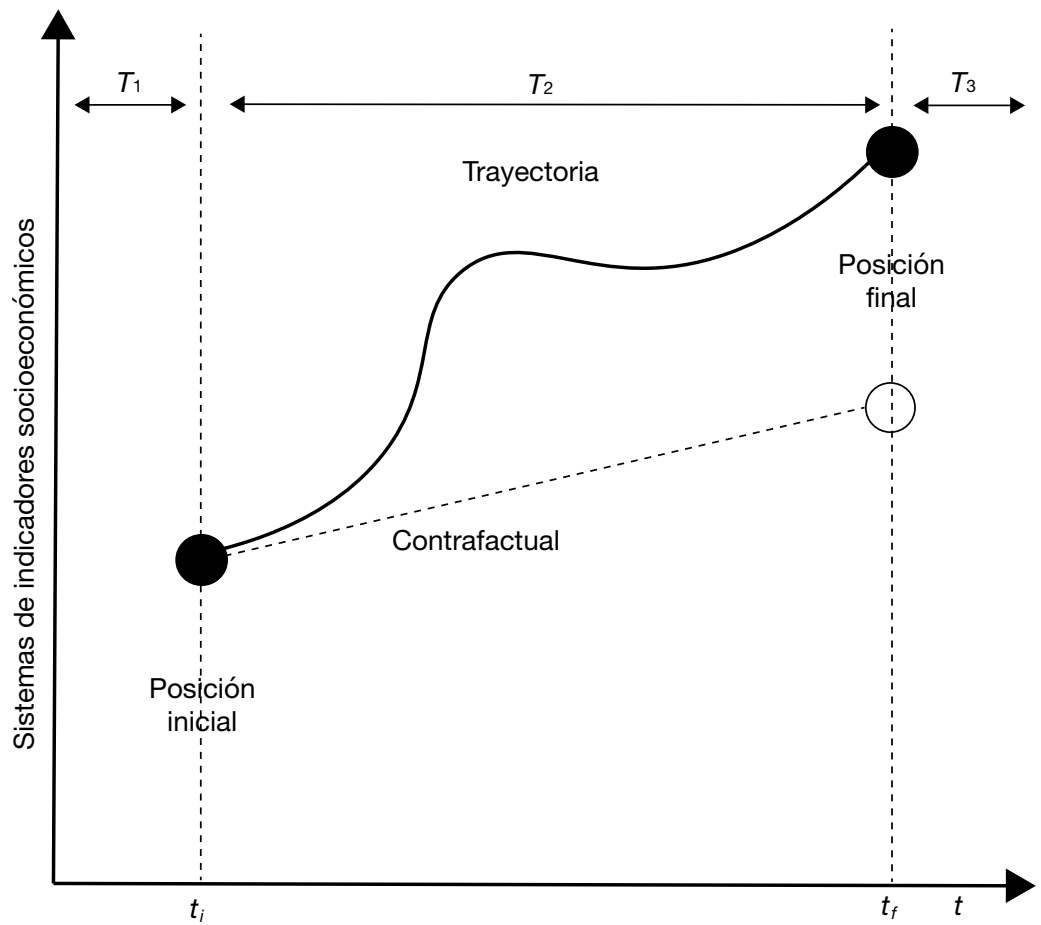

FUENTE: Elaboración propia.

Estos elementos básicos a su vez nos permiten diferenciar tres periodos de tiempo $\left(T_{1}, T_{2}\right.$ y $\left.T_{3}\right)$ en los que habitualmente se acometen las evaluaciones de las políticas públicas, ya sean ex-ante, intermedia, ex-post o continua. De este modo los resultados de los indicadores seleccionados nos permiten disponer de una medida del fenómeno estudiado y nos revelan posiciones relativas, cambios o tendencias, lo que además puede compararse con una situación contrafactual que podría haber sucedido en caso de que no se hubiera aplicado la actuación pública (Loi \& Rodrigues, 2012). Esto permite, de forma restrospectiva, abordar análisis situacionales (¿qué pasó?) o de causa-efecto (¿por qué pasó? ¿cuál fue la causa más probable?) así como, de forma prospectiva, diferentes estudios predictivos (¿qué pasará?) o prescriptivos (¿qué pasaría?). Claramente cada proceso de evaluación queda condicionado por la cantidad de datos -y microdatos- disponibles, los periodos de referencia del conjunto de indicadores utilizado y, de forma más general, con el grado de calidad de la información que proporcionan. 
En nuestro país la estadística oficial tiene una tradición muy amplia que se remonta al año 1856, con la creación de la Comisión de Estadística del Reino (Merediz, 2004), si bien con anterioridad se habían elaborado distintos trabajos censales de gran interés como el censo de pecheros de 1528, el censo de la Corona de Castilla de 1591 o el censo de Floridablanca de 1787. En sus orígenes la estadística estaba más estrechamente vinculada con los asuntos del Estado (Barreto-Villanueva, 2012) como, por ejemplo, el pago de impuestos, el reclutamiento de soldados o el reparto de tierras u otros bienes. Actualmente la producción de estadísticas se basa en una serie de principios (Eurostat, 2018) establecidos en el Código Europeo de Buenas Prácticas (CEBP), que proporcionan un sello de calidad único que facilita que la estadística mantenga un alto grado de credibilidad y confianza por parte de los usuarios. Estos conjuntos de principios de la estadística oficial se muestran en el Cuadro 1.

El grado de cumplimiento de todos esos principios tiene, sin duda, efectos en la realización de evaluaciones de políticas públicas. Así, desde una óptica temporal, la pertinencia, oportunidad, puntualidad, coherencia y comparabilidad de resultados constituyen una serie de propiedades requeridas a los indicadores estadísticos que tengan que ser utilizados en la evaluación. Por su parte, desde una óptica de las fuentes de datos, resulta necesario que estas sean independientes, confiables, basadas en una metodología sólida, con procedimientos estadísticos adecuados, que eviten una carga excesiva para las unidades informantes y sean rentables en el sentido de acometer un uso eficiente de los recursos. Desde el punto de vista de la política que se pretenda explicar, resulta especialmente significativo que las estadísticas se elaboren con una frecuencia y periodicidad suficiente, aportando además un adecuado nivel de desagregación.

En España los datos estadísticos oficiales constituyen un input necesario y fundamental para la evaluación de políticas públicas de organismos como la Autoridad Independiente de Responsabilidad Fiscal (AIReF, 2020a) o el Instituto para la

\section{CUADRO 1}

PRINCIPIOS DEL CEBP APLICADOS A LA ESTADÍSTICA OFICIAL DE ESPAÑA

\begin{tabular}{|c|c|c|}
\hline Entorno institucional & Procesos estadísticos & Producción estadística \\
\hline $\begin{array}{l}\text { 1. Independencia profesional. } \\
\text { 1. (bis). Coordinación y coope- } \\
\text { ración. } \\
\text { 2. Mandato de recogida de datos } \\
\text { y acceso a los datos. } \\
\text { 3. Adecuación de los recursos. } \\
\text { 4. Compromiso de calidad. } \\
\text { 5. Confidencialidad estadística } \\
\text { y protección de datos. } \\
\text { 6. Imparcialidad y objetividad. }\end{array}$ & $\begin{array}{l}\text { 7. Metodología sólida. } \\
\text { 8. Procedimientos estadísticos } \\
\text { adecuados. } \\
\text { 9. Carga no excesiva para los } \\
\text { encuestados. } \\
\text { 10. Rentabilidad. }\end{array}$ & $\begin{array}{l}\text { 11. Pertinencia. } \\
\text { 12. Precisión y fiabilidad. } \\
\text { 13. Oportunidad y puntualidad. } \\
\text { 14. Coherencia y comparabili- } \\
\text { dad. } \\
\text { 15. Accesibilidad y claridad. }\end{array}$ \\
\hline
\end{tabular}

FUENTE: Instituto Nacional de Estadística (INE) y elaboración propia. 
Evaluación de Políticas Públicas (Rico, 2020). La estadística oficial española se regula por medio de la Ley 12/1989, de 9 de mayo, de la Función Estadística Pública (LFEP), que normaliza la función estadística para fines estatales, la planificación y elaboración de las estadísticas desarrolladas por la Administración del Estado, y la organización de sus servicios estadísticos y sus relaciones en materia estadística con las comunidades autónomas, las corporaciones locales, la Unión Europea y los organismos internacionales. La LFEP establece el Plan Estadístico Nacional (PEN) como el principal instrumento ordenador de la actividad estadística de la Administración General del Estado. El PEN contiene las estadísticas que han de elaborarse a lo largo de un cuatrienio por los servicios estadísticos del Estado y las que han de llevarse a término, total o parcialmente, con participación de las comunidades autónomas y las corporaciones locales en virtud de acuerdos de cooperación o, en su caso, en ejecución de lo previsto en las leyes. Todas las estadísticas incluidas en el Plan tienen la consideración de estadísticas para fines estatales, se implementan mediante Programas anuales de desarrollo y son de cumplimentación obligatoria.

Si bien la ley estadística de 1989 ha permitido gobernar eficazmente la estadística oficial durante más de treinta años, actualmente existe un amplio consenso en que su revisión resultaría conveniente para poder adaptarla a las nuevas posibilidades de información que ofrece el actual proceso de transformación digital (Consejo Superior de Estadística, 2018).

\section{Estadísticas y evaluación de las políticas públicas en el contexto europeo}

En la Unión Europea (UE) las evaluaciones de políticas públicas de la Comisión siguen los principios de la iniciativa Better Regulation (Listorti et al., 2020). La agenda de Better Regulation está orientada hacia una evaluación sistemática del impacto económico, social y ambiental de la acción política para, así, tratar de asegurar que la legislación propuesta sea del más alto nivel. Un pilar para conseguir una mejor regulación es la evidencia empírica, que incluye la información cuantitativa y cualitativa proporcionada por la estadística oficial como elemento clave para describir el problema, comprender mejor su causalidad y, consecuentemente, la lógica de la intervención pública, y finalmente poder evaluar el impacto alcanzado. Por tanto, desde la óptica de la UE resulta fundamental que las estadísticas europeas dispongan de un fuerte grado de armonización y comparabilidad, tanto en la dimensión temporal (años, trimestres, meses) como espacial (estados miembros, regiones, ciudades), lo que a su vez permite y facilita la construcción de agregados europeos.

La primera «ley de estadística europea» (CE, 1997) se aprobó poco después de la inclusión del artículo 213-A en el Tratado de Ámsterdam, que habilitaba la elaboración de estadísticas comunitarias «cuando sean necesarias para la realización de las actividades de la Comunidad». Esta primera ley estadística fue remplazada, doce años después, por un nuevo reglamento sobre estadísticas europeas (CE, 2009) que sería revisado más tarde, en el año 2015. Todas estas normas han habilitado el desarrollo 
de diversos reglamentos estadísticos sectoriales, que garantizan una producción estadística armonizada en múltiples áreas temáticas, entre otras, las estadísticas coyunturales y estructurales de empresas, las estadísticas de precios y mercado laboral, las estadísticas sociales, o las estadísticas de agricultura y medio ambiente. También han facilitado la adopción de los sistemas contables europeos -incluida la contabilidad nacional y las cuentas satélite- y de varios conjuntos de indicadores europeos (Euroindicadores, Objetivos de Desarrollo Sostenible, Pilar Europeo de los Derechos Sociales, Economía Circular y Procedimiento de Desequilibrio Macroeconómico) para monitorizar distintas políticas públicas conforme se reflejan en el Cuadro 2.

\section{CUADRO 2}

SISTEMAS DE INDICADORES EN EL ÁMBITO DE LAS ESTADÍSTICAS EUROPEAS

\begin{tabular}{|c|c|}
\hline Conjunto de indicadores & Ámbitos \\
\hline Euroindicadores & $\begin{array}{l}\text { - Balanza de pagos. } \\
\text { - Encuestas a empresas y consumidores. } \\
\text { - Industria y servicios. } \\
\text { - Comercio internacional. } \\
\text { - Mercado laboral. } \\
\text { - Indicadores monetarios y financieros. } \\
\text { - Cuentas nacionales. } \\
\text { - Precios. }\end{array}$ \\
\hline $\begin{array}{l}\text { Objetivos de desarrollo } \\
\text { sostenible }\end{array}$ & $\begin{array}{l}\text { - Fin de la pobreza. } \\
\text { - Hambre cero. } \\
\text { - Salud y bienestar. } \\
\text { - Educación de calidad. } \\
\text { - Igualdad de género. } \\
\text { - Agua limpia y saneamiento. } \\
\text { - Energía asequible y no contaminante. } \\
\text { - Industria, innovación e infraestructura. } \\
\text { - Reducción de las desigualdades. } \\
\text { - Ciudades y comunidades sostenibles. } \\
\text { - Producción y consumo responsables. } \\
\text { - Acción por el clima. } \\
\text { - Vida submarina. } \\
\text { - Vida de ecosistemas terrestres. } \\
\text { - Paz, justicia e instituciones sólidas. } \\
\text { - Alianzas para lograr los objetivos. }\end{array}$ \\
\hline $\begin{array}{l}\text { Pilar europeo de derechos } \\
\text { sociales }\end{array}$ & $\begin{array}{l}\text { - Igualdad de oportunidades y acceso al mercado laboral. } \\
\text { - Mercados laborales dinámicos y condiciones laborales justas. } \\
\text { - Apoyo de las autoridades públicas / Protección e inclusión social. }\end{array}$ \\
\hline Economía circular & $\begin{array}{l}\text { - Producción y consumo. } \\
\text { - Gestión de residuos. } \\
\text { - Materias primas secundarias. } \\
\text { - Competitividad e innovación. }\end{array}$ \\
\hline $\begin{array}{l}\text { Procedimiento } \\
\text { de desequilibrio } \\
\text { macroeconómico }\end{array}$ & $\begin{array}{l}\text { - Desequilibrios externos y competitividad } \\
\text { - Desequilibrios internos. } \\
\text { - Indicadores de empleo }\end{array}$ \\
\hline
\end{tabular}

FUENTE: Eurostat y elaboración propia. 
Las estadísticas europeas, como servicio público, también son objeto de evaluaciones periódicas que refuerzan el fuerte compromiso existente con la calidad de los datos de los servicios estadísticos. Así en el Sistema Estadístico Europeo (SEE) se han definido cuatro niveles de garantía de calidad: el primero, formado por el conjunto de dieciséis principios recogidos en el Código Europeo de Buenas Prácticas; el segundo consiste en un conjunto de ochenta y cuatro indicadores relativos a cada uno de los principios anteriores; el tercero se basa en el denominado Marco de garantía de calidad del SEE (Eurostat, 2012), que proporciona diversos métodos y herramientas para facilitar la implementación práctica del Código de prácticas; en el cuarto nivel la calidad de los procesos y productos estadísticos individuales se evalúa en función de una serie de reglas estandarizadas e informes estructurados (Eurostat, 2020), que proporcionan a los usuarios información relevante sobre los métodos de recopilación y validación de datos tanto a nivel nacional como europeo. Estos cuatro niveles de garantía de la calidad se complementan con evaluaciones externas que, entre otros, incluyen un análisis periódico del grado de cumplimiento del Código en cada estado miembro por parte de Eurostat mediante los denominados Peer Reviews, los ejercicios de verificación de la Comisión Europea, u otras actuaciones específicas realizadas por terceros como, por ejemplo, el Tribunal de Cuentas Europeo (ECA, 2021).

La adopción del Reglamento 223/2009 sobre estadísticas europeas supuso la aprobación de provisiones específicas y novedosas que han permitido mejorar la calidad de los servicios proporcionados por la estadística oficial. En este punto resulta conveniente destacar dos de ellas establecidas en los artículos 17 bis y 23 de este Reglamento, respectivamente:

a) Las autoridades nacionales estadísticas «tendrán derecho a acceder a todos los registros administrativos sin demora y gratuitamente, a hacer uso de ellos y a integrarlos en las estadísticas en la medida necesaria para el desarrollo, la elaboración y la difusión de las estadísticas europeas». Asimismo se consultará sobre «el diseño inicial, el desarrollo posterior y la supresión de registros administrativos creados y mantenidos por otros organismos». Además, el Instituto Nacional de Estadística (INE) deberá «participar en las actividades de estandarización relativas a registros administrativos que sean de utilidad para la generación de estadísticas europeas».

b) Eurostat y los institutos nacionales de estadística «en sus respectivas esferas de competencia, podrán conceder el acceso a datos confidenciales que solo permitan la identificación indirecta de unidades estadísticas a investigadores que lleven a cabo análisis estadísticos con fines científicos», dando de este modo respuesta a la fuerte demanda existente para el acceso a los microdatos de las operaciones estadísticas (Crato \& Paruolo, 2019). 
Sin duda ambas medidas han supuesto un paso importante en la dirección de mejorar la calidad de las estadísticas, facilitando un uso más intensivo de los recursos estadísticos por parte de investigadores y salvaguardando en todo caso la confidencialidad y el secreto estadístico. Sin embargo, la legislación estadística de los países de la Unión Europea en general no contiene provisiones directas sobre evaluación de políticas públicas si bien podemos encontrar algunas referencias indirectas que podrían ser entendidas como una finalidad propia de las estadísticas. A modo de ejemplo, en Países Bajos se atribuye al Central Bureau of Statistics la misión de realizar actividades estadísticas con fines de investigación y publicar las estadísticas compiladas sobre la base de dicha investigación; por su parte la ley estadística de Letonia incluye entre los objetivos de la estadística oficial el monitoreo o evaluación de leyes y reglamentos o documentos de planificación del desarrollo, e incluso la redacción de los escenarios de previsiones; en Rumanía, su ley establece que la estadística oficial facilita la formulación, implementación, modernización y evaluación de políticas nacionales, planes de desarrollo nacionales y locales, de conformidad con los principios fundamentales de las estadísticas oficiales; y en Reino Unido, el Statistics Board tiene atribuida la función de apoyar en el desarrollo de evaluación de políticas públicas. Precisamente este último es el enfoque que se sigue en la ley genérica de estadística, que señala que debería atribuirse al consejo estadístico la tarea de «promover el uso de estadísticas oficiales como evidencia para la formulación de políticas públicas, su evaluación y toma de decisiones» (UNECE, 2018).

Por otra parte, las oficinas de estadísticas europeas son conscientes de las posibles soluciones que aportan los nuevos roles de administración y gestión de datos, de forma que se están produciendo adaptaciones en las funciones desempeñadas hasta ahora. Así, el Instituto Nacional de Estadística de Italia cuenta desde diciembre 2020 con el «Centro ISTAT para estadísticas inteligentes confiables» (Istat Centre for Trusted Smart Statistics) destinado a consolidar y desarrollar la experiencia interna en la gestión del big data, incluido el apoyo de la estadística oficial a cuestiones políticas de nivel gubernamental. En Lituania, tras la reciente reforma de su ley de estadística en octubre de 2020, la oficina de estadística cuenta con dos nuevas funciones: a) establecer el «Sistema de Información de Gobernanza de Datos Estatales»; b) gestionar la información contenida en el sistema de información del Estado. Por su parte, la Oficina Federal de Estadística de Suiza ejerce el liderazgo en la «Gestión nacional de datos» (Nationale Datenbewirtschaftung, $N a D B$ ) a través de una plataforma de interoperabilidad en la que se integran datos administrativos del gobierno central, de los cantones y de empresas privadas.

Situándonos en un marco geográfico más amplio, en Estados Unidos el acceso directo y seguro a los microdatos administrativos también se ha considerado una prioridad (Card et al., 2010). En cuanto a la Organización para la Cooperación y Desarrollo Económicos (OCDE) la estadística también constituye un elemento fundamental a la hora de elaborar mejores políticas para mejores vidas (better policies for better lives) así como para proceder a su evaluación (OCDE, 2020b). Esta organi- 
zación internacional ha sido clave en el desarrollo de diversos productos estadísticos orientados para la evaluación de políticas públicas, entre otros, el Manual de Frascati para encuestas de I+D, las pruebas del programa para la evaluación internacional de alumnos (PISA) o el índice de calidad de vida de la iniciativa «How's life?» (OCDE, 2020a). Conforme se observa en la Figura 3, esta iniciativa proporciona estimaciones de bienestar nacional y regional considerando hasta once dimensiones diferentes: vivienda, ingresos, empleo, comunidad, educación, medio ambiente, compromiso cívico, salud, satisfacción, seguridad, balance vida-trabajo, lo que permite la realización de comparaciones transnacionales.

\section{FIGURA 3}

ESTIMACIÓN DEL BIENESTAR REGIONAL

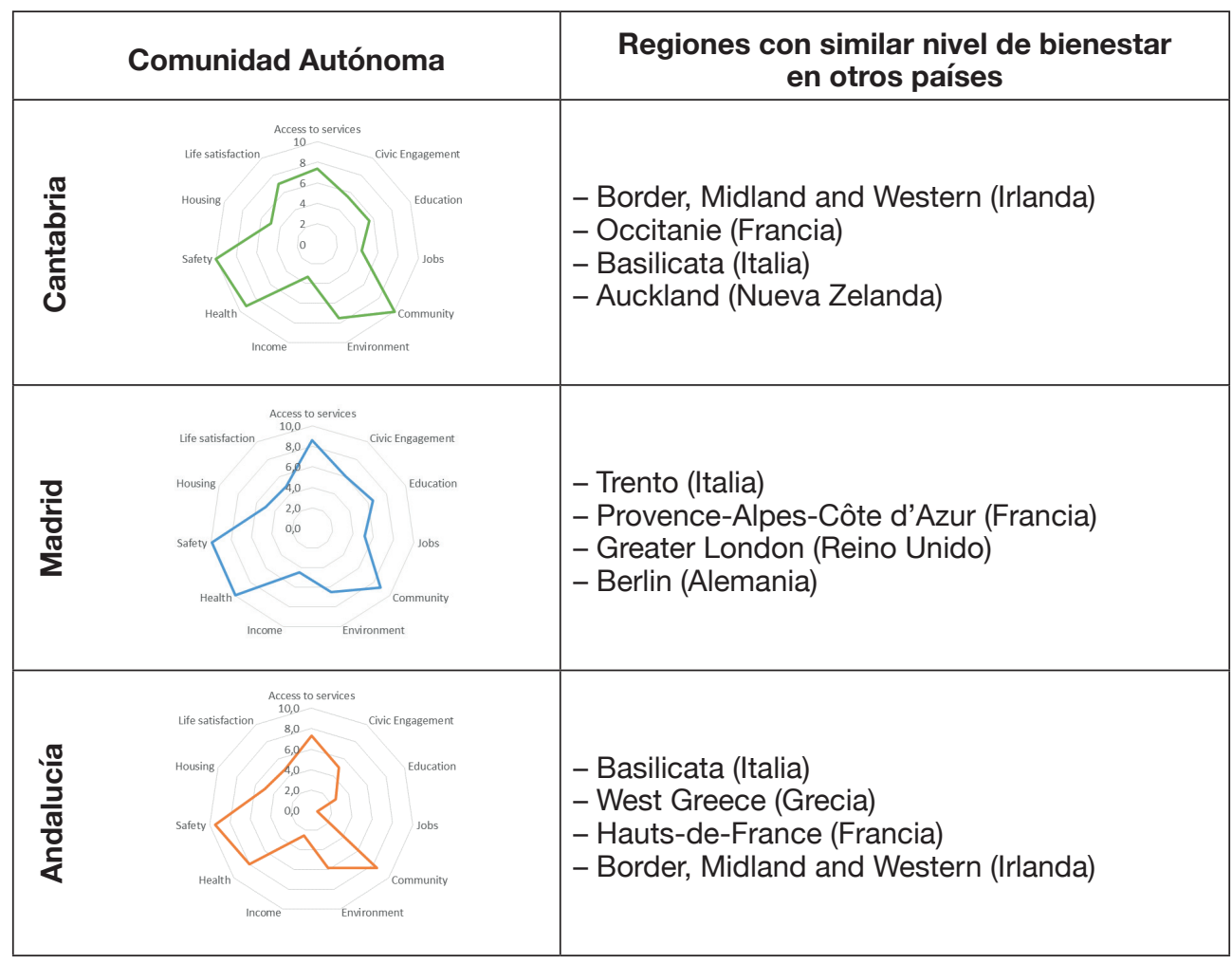

FUENTE: OCDE regional well-being. 


\section{Hacia un nuevo paradigma de datos e información estadística para la evaluación de políticas públicas}

En la actualidad vivimos en una sociedad altamente datificada que se caracteriza por la existencia de nuevos y variados ecosistemas de datos, que constituyen en sí una clara oportunidad para que la estadística oficial refuerce su estrecho vínculo con la evaluación de políticas públicas, aportando mucha más información puntual y granular. En efecto, el acceso a nuevas fuentes de datos permite avanzar en la puesta en marcha de nuevos productos estadísticos que, utilizando metodologías innovadoras, facilita dar respuestas a las necesidades de los investigadores y evaluadores de políticas públicas ofreciéndoles un desglose de datos mucho más detallado e incrementando la frecuencia de la disponibilidad de la información, es decir, nuevas estadísticas en alta definición y tiempo real.

Un claro ejemplo de esta nueva situación lo constituyen las estadísticas experimentales que han puesto en marcha los sistemas estadísticos nacional y europeo para, así, dar respuesta de manera más oportuna a las necesidades de los usuarios. Si bien estas estadísticas no necesariamente cumplen por el momento con todos los requisitos de calidad y grado de armonización exigibles a las estadísticas oficiales, su utilidad es manifiesta al cubrir claros vacíos de información estadística, facilitando el diseño de nuevos indicadores y proporcionando nueva información para el diseño y evaluación de políticas públicas. Existen varios prototipos de estadísticas experimentales, entre los cuales se pueden citar: la estimación del número de defunciones semanales durante el brote de la COVID-19, la estimación mensual de nacimientos; la coyuntura demográfica de empresas, el atlas de distribución de renta de los hogares, los estudios de movilidad a partir de la telefonía móvil, la medición del comercio diario al por menor de grandes empresas, el gasto realizado por los visitantes extranjeros en sus visitas a España, la medición del número de viviendas turísticas en España y su capacidad, o la medición del turismo nacional y receptor a partir de la posición de los teléfonos móviles. En este punto es necesario puntualizar que estas estadísticas experimentales no son exclusivas del ámbito nacional o europeo, sino que también han proliferado en otras zonas, por ejemplo, en Estados Unidos donde el Census Bureau ha puesto en marcha nuevas estadísticas como el Census Household Pulse Survey o el Small Business Pulse Survey.

A modo de ejemplo, la Figura 5 muestra la variación mensual de los nacimientos con respecto a 2019 del acumulado en lo que va de año, revelando un notable retroceso de esta variable en enero de 2021 con un descenso en el número de nacimientos del $22,84 \%$, que paulatinamente ha ido recuperándose hasta situarse en un $-9,59 \%$ en el mes de abril de 2021. Este gráfico también nos aporta el exceso de mortalidad en España, elaborado con periodicidad mensual y en comparación con la media mensual obtenida durante el periodo 2016-2019. El valor negativo obtenido en enero, febrero, junio de 2020 y marzo de 2021 nos indica un menor número de fallecidos durante esos meses comparados con el periodo base, en tanto que el máximo de la serie se obtiene en los meses de abril, marzo y noviembre de 2020. 
FIGURA 5

\section{VARIACIÓN DE LA NATALIDAD Y EL EXCESO DE MORTALIDAD EN 2020 Y 2021 POR MESES}

(En \%)

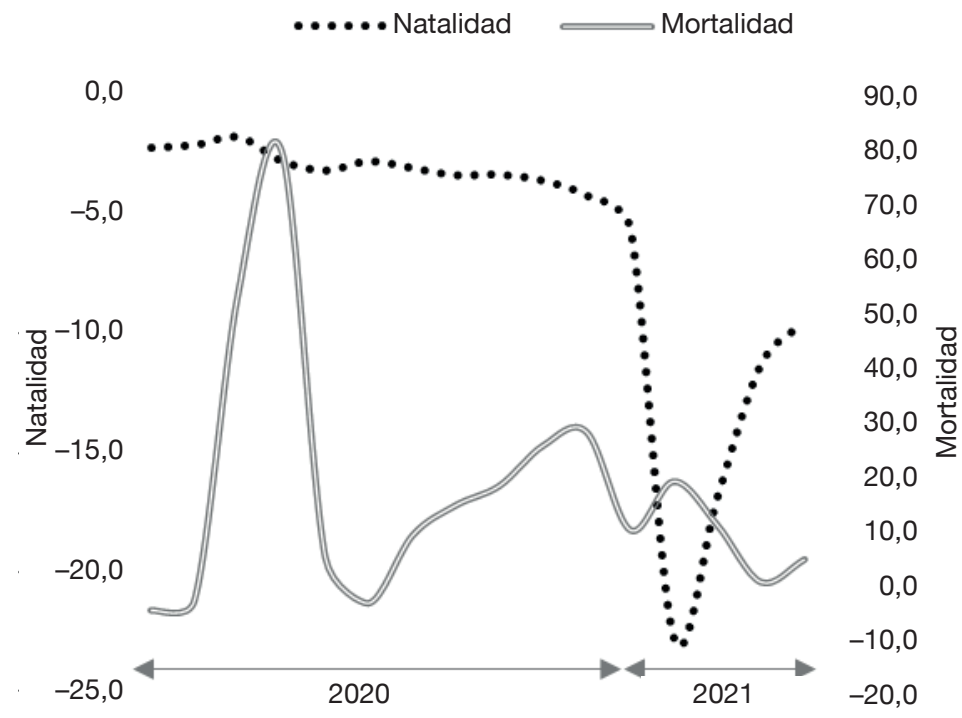

FUENTE: INE y elaboración propia.

Sin duda, las estadísticas experimentales anteriormente mencionadas amplían el sistema de información estadístico existente y han sido muy bien acogidas por los usuarios. No obstante esta medida también puede ser complementada por otras actuaciones que les permitan trabajar directamente con las bases de datos, para realizar investigaciones y estudios de interés público salvaguardando en todo caso el secreto estadístico, la confidencialidad y la protección de los datos. En este sentido, el reciente acuerdo alcanzado en abril de 2021 entre el INE, la Agencia Estatal de Administración Tributaria, la Seguridad Social, el Servicio Público de Empleo Estatal y el Banco de España permitirá incrementar la utilidad de la información administrativa disponible así como de los datos recogidos con fines estadísticos, habilitando el acceso de datos granulares a investigadores que desarrollen proyectos científicos de interés público. Se trata de un enfoque que da respuesta a muchas demandas de investigadores y organismos públicos, entre otros, al planteamiento realizado por la AIReF en su opinión para una estrategia de acceso a datos administrativos (AIReF, 2020b). No obstante, la puesta en marcha de un proyecto de este calibre requiere nuevas inversiones para actualizar las infraestructuras tecnológicas así como una adaptación del marco normativo existente, particularmente, la Ley de la Función Estadística Pública, para permitir un acceso a datos por parte de los investigadores, al menos, en las mismas condiciones al modelo ya establecido en el ámbito de la ley estadística europea. 
Un caso evidente de creación de infraestructura tecnológica sería la implantación de un nodo de interoperabilidad que permita el intercambio de información entre los agentes implicados que se determinen. La información a intercambiar podría consistir en datos y ficheros procedentes de encuestas, de registros administrativos o de otras fuentes alternativas, en tanto que el conjunto de agentes implicados podrían ser desde los servicios estadísticos (estatales o autonómicos), servicios públicos no estadísticos, investigadores o entidades de investigación de diferente naturaleza jurídica. En este punto es necesario subrayar que los usos que se podrían hacer de dicha información deberían ser diferentes en función de las necesidades de los distintos y potenciales agentes mencionados anteriormente. Y, en todo caso, cualquier acceso y posible uso tendría que estar regulado por la legislación y ser objeto de la correspondiente monitorización. La disponibilidad de un sistema de integración y acceso a datos, tanto estadísticos como administrativos, que permita el acceso a los mismos de forma eficiente y con las condiciones de seguridad convenientes, nos permitiría alinearnos con las iniciativas más punteras que están surgiendo a nivel internacional para facilitar una mayor interoperabilidad de datos e información estadística.

Junto con la inversión tecnológica también resulta preciso adaptar los conocimientos del personal para poder afrontar una producción estadística basada en múltiples fuentes de información, favoreciendo la incorporación de profesionales con formación orientada a los métodos y herramientas para el análisis de datos masivos (estadísticos especializados en ciencia de datos) y, al mismo tiempo, de técnicos con amplios conocimientos de índole contable y demográfica (estadística aplicada a las ciencias económicas y sociales). Esta medida puede venir complementada con otras actuaciones para reforzar la colaboración con las universidades, promoviendo la realización de proyectos de investigación científica que permitan el desarrollo de nuevos conocimientos y mejoren la producción de datos y estadísticas contribuyendo, además, a la I+D+i en estadística en áreas clave como pueden ser: la codificación automática con técnicas de machine learning, el lenguaje natural e inteligencia artificial aplicados a la recogida de datos, la publicación y uso de datos abiertos enlazados (linked open data), o la aplicación de estimación en pequeñas áreas para el uso de información auxiliar en la desagregación de la información de encuestas, entre otros. Es necesario apuntar que este nuevo paradigma de datos e información estadística se encuentra esbozado en las propias líneas estratégicas del Programa anual 2021 del Plan Estadístico Nacional 2021-2024, aprobado por medio del Real Decreto 150/2021 de 9 de marzo, que entre otros incluye el incremento de la explotación de los registros administrativos en la producción de estadísticas oficiales; el establecimiento de una arquitectura de datos comunes procedentes de registros administrativos asociados a los principales directorios con un tratamiento armonizado; inclusión de identificadores estandarizados; el análisis de la creación de plataformas seguras para almacenar registros administrativos para su posterior explotación estadística; o la creación de un Sistema integrado de Información de las Empresas.

Para concluir, debemos tener presente los notables avances que la Unión Europea está realizando en materia de digitalización y, particularmente, el impulso que se está 
otorgando tanto a la creación de un espacio común europeo de datos así como a la reutilización de información del sector público. En efecto, la estrategia de datos europea facilita un marco de gobernanza intersectorial para el acceso a los datos y su uso, además de la creación de espacios comunes de datos en sectores estratégicos y de interés público. En este contexto resulta conveniente que el sistema estadístico pueda desarrollar funciones de administrador de datos (data stewardship) y participe en el diseño de una estructura de gobernanza que facilite el intercambio de datos Business to Government (B2G). De hecho países como Canadá, Estonia, Irlanda, Reino Unido o Nueva Zelanda son punteros a la hora de buscar sinergias entre los roles de Chief Statistician y Chief Data Steward. Una situación en la que los servicios estadísticos puedan actuar como data steward ofrecería múltiples oportunidades para la producción estadística oficial, al permitir la utilización de nuevas fuentes de información en la producción estadística, reduciendo el coste y carga de las unidades informantes al combinar datos estadísticos con registros administrativos y otras fuentes de datos privadas, permitiendo a su vez mejorar la utilidad social de los datos, en particular para la evaluación de políticas públicas, que pueden beneficiarse de la contrastada experiencia del INE en el tratamiento y normalización de los datos. Por ello resulta conveniente avanzar en el diseño de una «estrategia estadística del dato» que consiga integrar las perspectivas Government to Government $(G 2 G)$, Government to Business $(G 2 B)$ y Business to Government $(B 2 G)$ y fomente en particular el acceso a los datos para investigación (Consejo Superior de Estadística, 2021). En particular, el próximo Data Act que se aprobará en el seno de la Unión Europea proporcionará una oportunidad única para promover la compartición de datos $B 2 G$ para fines de interés público, en especial para la estadística oficial (es decir, B2G4S) manteniendo en todo caso las condiciones de salvaguarda, confidencialidad y secreto estadístico de la información.

\section{Conclusiones}

Una estadística oficial de calidad constituye un elemento esencial para el proceso de toma de decisiones y la evaluación de políticas públicas. Durante décadas los servicios estadísticos han cumplido su misión con éxito utilizando información procedente de las fuentes de datos tradicionales (censos, encuestas y registros administrativos) si bien este modelo ya no resulta del todo suficiente. La senda de modernización de productos y procesos estadísticos ha estado principalmente orientada a incrementar tanto la elaboración de nuevas estadísticas como el grado de satisfacción de los usuarios. La crisis de la COVID-19 ha demostrado la resiliencia del INE y de la estadística oficial en su conjunto, que están sólidamente fundamentados, si bien aún quedan cuestiones por resolver particularmente en lo que respecta al uso del big data en la estadística oficial.

La utilización de estas nuevas fuentes de datos en la estadística oficial conlleva la necesidad de adaptar los enfoques de la producción estadística, las capacidades y 
competencias de los estadísticos, los métodos de producción y las tecnologías utilizadas, buscando que nuestras estadísticas sigan siendo relevantes y significativas, y manteniendo en todo caso el alto grado de confianza que tiene la sociedad en los servicios estadísticos. Para ello es necesario poder acceder a las nuevas fuentes de datos e integrarlas junto con las otras fuentes de datos más tradicionales para la producción de estadísticas oficiales. Es necesario también disponer de recursos humanos y tecnológicos suficientes, incluyendo procesos, estándares y metodologías armonizadas a nivel europeo y, todo ello, dentro de un marco de preservación de la privacidad y con la máxima garantía en la seguridad de los datos.

Para superar exitosamente estos retos es necesario, en primer lugar, adaptar la legislación nacional existente. Las nuevas reglas deben tener como objetivo maximizar el beneficio para toda la sociedad equilibrando los derechos e intereses legítimos de todas las partes interesadas involucradas. El equilibrio de intereses y derechos debe basarse, por un lado, en el derecho de los ciudadanos a la privacidad individual como sujetos de datos y a vivir en una sociedad democrática informada como usuarios de estadísticas y, por otro lado, en el respeto a los intereses comerciales de las empresas que custodian los datos así como los intereses comerciales de todos los demás actores económicos para tomar sus decisiones informadas basadas en estadísticas públicas. Varias actuaciones se han puesto en marcha en paralelo para hacer frente a esta situación: a nivel europeo se están tomando iniciativas para que las necesidades estadísticas estén convenientemente recogidas en la futura Ley del Dato Europea (Data Act) y, en caso de ser necesario, en la ley estadística europea; y a nivel nacional el Plan Estadístico Nacional ya contempla como una de las líneas estratégicas para este periodo la reforma de nuestra ley estadística, la Ley de la Función Estadística Pública, en particular para recoger estos aspectos anteriormente señalados.

En segundo lugar, como ya se ha mencionado, es necesario abordar la modernización de la producción estadística, en especial las infraestructuras tecnológicas que favorezcan la interoperabilidad de los datos entre el conjunto de agentes que se determinen. Adicionalmente es necesario adecuar los conocimientos y capacidades del personal incluyendo una especialización y adaptación de las pruebas de acceso a los cuerpos de estadística del estado, potenciando nuevos cursos de formación, el uso de nuevas herramientas dirigidas a alcanzar este objetivo así como la colaboración con el mundo académico y universitario.

Por último, es importante que en todo momento quede garantizado el mismo nivel de armonización y alta calidad de las estadísticas basadas en big data en relación con el que gozan las estadísticas anteriormente calificadas como tradicionales. Para ello se necesita seguir participando en los estudios piloto a nivel europeo en los que se testan nuevas metodologías, a la vez que continuar avanzando en esta línea con las estadísticas experimentales, y buscar la colaboración de los medios académicos y del sector privado, para avanzar conjuntamente y alcanzar el objetivo común que no es otro que la mejora de nuestra sociedad. 


\section{Referencias bibliográficas}

AIReF, Autoridad Independiente de Responsabilidad Fiscal (2020a). Estudio de Beneficios Fiscales de la Fase II del Spending Review. https://www.airef.es/wp-content/ uploads/2020/10/Docus_Varios_SR/Estudio_Beneficios_Fiscales_Spending_Review.pdf

AIReF, Autoridad Independiente de Responsabilidad Fiscal (2020b). Opinión para una estrategia de acceso a datos administrativos. https:/www.airef.es/wp-content/uploads/2020/ 10/OPINION_DATOS/201005.-Opinion-Acceso-Datos.pdf

Arnaboldi, M., \& Azzone, G. (2020). Data science in the design of public policies: Dispelling the obscurity in matching policy demand and data offer. Heliyon, 6(6), e04300. https://doi.org/10.1016/j.heliyon.2020.e04300

Azzone, G. (2018). Big data and public policies: Opportunities and challenges. Statistics \& Probability Letters, 136, 116-120. https://doi.org/10.1016/j.spl.2018.02.022

Barreto-Villanueva, A. (2012). El progreso de la Estadística y su utilidad en la evaluación del desarrollo. Papeles de población, 18(73), 241-271.

Card, D., Chetty, R., Feldstein, M. S., \& Saez, E. (2010). Expanding access to administrative data for research in the United States. American Economic Association, ten years and beyond: Economists answer NSF's call for long-term research agendas. National Science Foundation

CE (1997). Reglamento (CE) no 322/97 del Consejo de 17 de febrero de 1997 sobre la estadística comunitaria. Diario Oficial de las Comunidades Europeas. https://eur-lex.europa.eu/legal-content/ES/TXT/?uri=CELEX:31997R0322

CE (2009). Reglamento (CE) no 223/2009 del Parlamento Europeo y del Consejo de 11 de marzo de 2009 relativo a la estadística europea. Diario Oficial de la Unión Europea. https://eur-lex.europa.eu/legal-content/EN/TXT/?uri=CELEX\%3A02009R0223-20150608

Consejo Superior de Estadística (2018). Propuestas y recomendaciones, previas a la formulación del anteproyecto del Plan Estadístico Nacional 2021-2024, sobre las necesidades nacionales en materia estadística y la adaptación y mejora de los medios existentes. https:// www.ine.es/normativa/leyes/plan/plan_2021-2024/recomendaciones_2021-2024_cse.pdf

Consejo Superior de Estadística (2021). El papel de la estadística oficial en la administración y gestión de datos. https://www.ine.es/normativa/leyes/cse/papel_estadistica_oficial.pdf

Crato, N., \& Paruolo, P. (2019). Data-Driven Policy Impact Evaluation: How Access to Microdata is Transforming Policy Design. Springer Open. https://doi.org/10.1007/978-3319-78461-8

ECA, European Court of Auditors. (2021, 4 de mayo). Los auditores estudian la calidad de las estadísticas de la UE. [comunicado de prensa] https://www.eca.europa.eu/Lists/ECADocuments/INAP21_05/INAP_High-quality-EU-stats_ES.pdf

Eurostat (2012). Quality Assurance Framework of the European Statistical System. https://ec.europa.eu/eurostat/documents/3859598/5923349/QAF_2012-EN.PDF.pdf/ fcdf3c44-8ab8-41b8-9fd0-91bd1299e3ef?t=1414781890000

Eurostat (2014). Towards a harmonised methodology for statistical indicators.Part 1, Indicator typologies and terminologies: 2014 edition. https://doi.org/10.2785/56118

Eurostat (2018). Código de buenas prácticas de las estadísticas europeas. https://doi.org/10.2785/232692

Eurostat (2020). European Statistical System (ESS) handbook for quality and metadata reports. https://doi.org/10.2785/666412 
Fano, M. P. (2017). Un nuevo marco legal para la evaluación. Gestión y análisis de políticas públicas, (17), 6-25. https://doi.org/10.24965/gapp.v0i17.10397

Feinstein, O. (2007). Evaluación pragmática de políticas públicas. ICE, Revista de Economía (836).

Ley 12/1989, de 9 de mayo, de la Función Estadística Pública. Boletín Oficial del Estado, n ${ }^{\circ}$ 112, de 11 de mayo de 1989, pp. 14026 a 14035. https://www.boe.es/eli/es/1/1989/05/09/12

Listorti, G., Basyte-Ferrari, E., Acs, S., \& Smits, P. (2020). Towards an Evidence-Based and Integrated Policy Cycle in the EU: A Review of the Debate on the Better Regulation Agenda. JCMS: Journal of Common Market Studies, 58(6), 1558-1577. https://doi.org/10.1111/jcms.13053

Loi, M., \& Rodrigues, M. (2012). A note on the impact evaluation of public policies: The counterfactual analysis. JRC. https://doi.org/10.2788/50327

Merediz, A. (2004). Historia de la estadística oficial como institución pública en España. Instituto de Estadística de Andalucía. https://www.juntadeandalucia.es/institutodeestadisticaycartografia/historicas/HistEstOficial/HistEstOficial.pdf

Moral, I., Paniagua, M., Rodríguez, L., \& Rodríguez, C. (2016). Evaluación de políticas públicas. Técnicas cuantitativas. Garceta

OCDE, Organización para la Cooperación y el Desarrollo Económico. (2020a). How's Life? Measuring Well-being. https://doi.org/10.1787/9870c393-en

OCDE, Organización para la Cooperación y el Desarrollo Económico. (2020b). Improving governance with policy evaluation: Lessons from country experiences. https://doi.org/10.1787/89b1577d-en

Pollitt, C., \& Bouckaert, G. (2010). La reforma de la gestión pública: Un análisis comparado. INAP.

Radermacher, W. J. (2019). Governing-by-the-numbers/Statistical governance: Reflections on the future of official statistics in a digital and globalised society. Statistical Journal of the IAOS, 35(4), 519-537. https://doi.org/10.3233/SJI-190562

Rico, J. (2020). Guía de evaluación de resultados de políticas públicas. Instituto para la Evaluación de Políticas Públicas. https:/www.mptfp.gob.es/dam/es/portal/funcionpublica/evaluacion-politicas-publicas/ Documentos/Metodologias/Guia_de_Evaluacion_de_Resultados.pdf\#page=1

Statistics Canada (2003). Survey methods and practices.

Tratado de Ámsterdam por el que se modifica el tratado de la Unión Europea, los tratados constitutivos de las Comunidades Europeas y determinados actos conexos. Diario Oficial de las Comunidades Europeas. C340, de 10 de noviembre de 1997. http://data.europa.eu/ eli/treaty/ams/sign

UNECE, United Nations Economic Commission for Europe. (2009). Cómo hacer comprensibles los datos. Parte 2: Una guía para presentar estadísticas. https://unece.org/DAM/stats/documents/writing/MDM_Part2_Spanish.pdf

UNECE, United Nations Economic Commission for Europe. (2018). Guidance on Modernizing Statistical Legislation.

https://unece.org/DAM/stats/publications/2018/ECECESSTAT20183.pdf 